\title{
Dosimétrie passive : introduction d'un nouveau dosimètre basé sur la technologie OSL
}

\author{
V. ARCHAMBAULT ${ }^{1}$, G. LE ROY ${ }^{1}$, B. PRUGNAUD ${ }^{1}$
}

(Manuscrit reçu le 25 août 2005, accepté le 29 août 2005)

RÉSUMÉ Un nouveau dosimètre passif basé sur la technologie OSL vient d'être introduit sur le marché français. Dans cet article sont décrits : la technologie et le matériau sur lequel s'appuie ce nouveau détecteur, le dosimètre lui-même.

ABSTRACT Passive dosimetry: introduction of a new dosimeter based on OSL technology.

A new passive dosimeter based on OSL technology has been introduced on the French market. In this article are described: the technology and the material on which this new detector relied, the dosimeter itself.

Keywords: dosimeter / dosimetry / radiation / radioprotection / OSL

\section{Introduction}

Jusqu'en 1999, les dosimètres photographiques étaient les détecteurs imposés par la réglementation française pour effectuer la surveillance dosimétrique externe des travailleurs susceptibles d'être exposés aux rayonnements ionisants. Un arrêté du 31 décembre 2004 abaisse le seuil de détection minimale des dosimètres ${ }^{2}$ à $0,1 \mathrm{mSv}$. Les dosimètres films ne répondant pas à ces critères pour certaines énergies, LCIE LANDAUER vient d'introduire sur le marché un nouveau dosimètre basé sur la technologie OSL (Optically Stimulated Luminescence ou luminescence stimulée optiquement) qui offre, entre autres, une meilleure sensibilité de détection.

\section{Présentation de la luminescence stimulée optiquement}

\subsection{La technologie : OSL}

L'OSL est une technologie utilisée à travers le monde depuis plusieurs années pour le suivi dosimétrique de plus de 1300000 personnes. L'OSL est aujourd'hui une

1 LCIE LANDAUER, 33 avenue du Général Leclerc, 92260 Fontenay-aux-Roses, France.

2 À titre transitoire, jusqu'au 30 décembre 2007, ce seuil est porté à $0,20 \mathrm{mSv}$. 
des techniques de dosimétrie passive reconnues par la réglementation avec le film photographique et le TLD (ThermoLuminescent Dosimeter ou luminescence stimulée thermiquement). À ce jour, LCIE LANDAUER est le seul laboratoire national agréé utilisant cette technologie.

Tout comme le TLD, la technologie OSL repose sur le principe de lecture d'une émission de lumière. Cette luminescence est provoquée par un flash lumineux - laser, diode électroluminescente (DEL) - dans le cas de l'OSL et par chauffage pour le TLD. Lors d'une interaction avec un rayonnement ionisant, des charges se retrouvent piégées dans le matériau sensible. Ces électrons sont retenus dans ces pièges jusqu'à ce qu'ils reçoivent une excitation extérieure qui les libèrent. Ces charges émettent alors une impulsion de lumière proportionnelle à la dose de rayonnement reçue.

Dans le cas de l'OSL, la source de stimulation utilisée est facilement contrôlable (intensité, durée). De ce fait, seulement une petite proportion des charges retenues dans le matériau sensible est libérée. Une très grande partie de l'information est conservée dans le capteur. Contrairement au TLD où les pièges sont entièrement vidés, la stimulation optique autorise la relecture du dosimètre. Pour en savoir plus, http://www.osldosimetry.com.

\subsection{Le matériau utilisé par LANDAUER : $\mathrm{Al}_{2} \mathrm{O}_{3}: \mathrm{C}$}

InLight $^{\circledR}$, le nouveau dosimètre passif lancé par LCIE LANDAUER - filiale européenne du groupe LANDAUER - repose sur la technologie OSL. La mesure de dose s'effectue grâce à une fine feuille de poudre d'oxyde d'aluminium dopé au carbone, $\mathrm{Al}_{2} \mathrm{O}_{3}: \mathrm{C}$. Cette poudre résulte d'un cristal obtenu après mélange à haute température d'oxyde d'aluminium pur et de dopants. Cette dernière est ensuite enduite grâce à un liant entre deux bandes de film polyester, l'ensemble formant ainsi un ruban. À noter, ce cristal est fabriqué par LANDAUER suivant des spécifications propres. 1500000 dosimètres peuvent être produits à partir d'un même cristal.

Pour chaque rouleau de ruban d'oxyde d'aluminium (Fig. 1), LANDAUER réalise des contrôles qualité afin de déterminer le facteur de sensibilité applicable à tous les détecteurs OSL qui seront issus de cette fabrication. La bande, d'une épaisseur de $0,3 \mathrm{~mm}$, est ensuite coupée à la taille et à la forme désirées suivant le type de détecteurs à produire. La sensibilité de chaque lot est gravée sur le porteécrans de chaque dosimètre sous forme d'un code 2D-Matrix. Contrairement au TLD qui nécessite de connaître le facteur de sensibilité de chaque carte, ce procédé autorise une analyse des détecteurs dans différents lecteurs sans nécessité de données supplémentaires. 


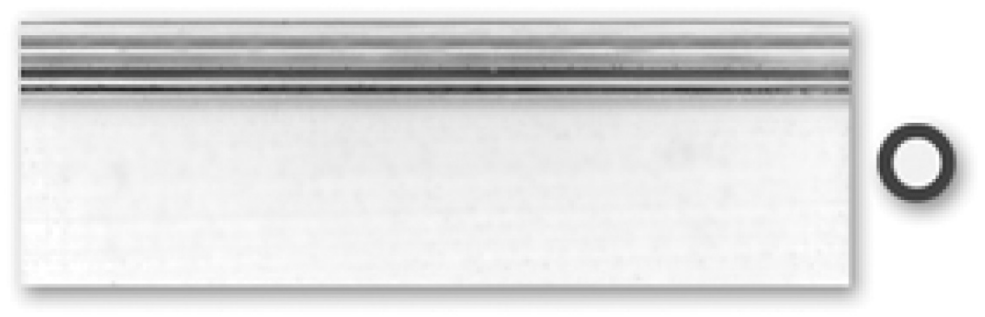

Figure 1 - Ruban d'oxyde d'aluminium.

Aluminium oxide strip.

LANDAUER a choisi d'employer l'oxyde d'aluminium dopé au carbone en raison de sa sensibilité 40 à 60 fois plus élevée que le fluorure de lithium FLi fréquemment utilisé pour les détecteurs TLD. Il offre également une sensibilité nettement meilleure que le film photographique. Ce matériau est capable de mesurer des doses allant de $0,01 \mathrm{mSv}$ à $10 \mathrm{~Sv}$ dans des gammes d'énergie de $5 \mathrm{keV}$ à $40 \mathrm{MeV}$ suivant le type de rayonnements ionisants. L'oxyde d'aluminium n'est affecté ni par la chaleur, ni par l'humidité. Il est insensible aux rayonnements neutroniques.

\section{Introduction d'un nouveau dosimètre passif, InLight ${ }^{\circledR}$}

\subsection{Le dosimètre InLight ${ }^{\circledR}$}

InLight ${ }^{\circledR}$ se présente sous la forme d'une coque en plastique dur et translucide dans laquelle s'insère un tiroir porte-élément et une étiquette d'information (Fig. 2).

Il se compose de quatre éléments détecteurs OSL de $5 \mathrm{~mm}$ de diamètre qui sont insérés dans une plaque munie de quatre trous. Cette plaque vient s'emboîter dans un porte-écrans équipé au recto et au verso de quatre filtres (Fig. 3) - fenêtre ouverte, cuivre, aluminium et plastique - qui se positionnent devant chacune des pastilles d' $\mathrm{Al}_{2} \mathrm{O}_{3}: \mathrm{C}$. Grâce à ce système, les détecteurs sont capables de mesurer et différencier les rayonnements $\mathrm{X}$, gamma et bêta quelque soit l'orientation du dosimètre. À noter, InLight ${ }^{\circledR}$ répond déjà aux dispositions de l'arrêté du 31 décembre 2004 qui abaisse le seuil de détection des dosimètres passifs à $0,10 \mathrm{mSv}$.

Outre le porte-écrans, le tiroir porte-éléments est conçu pour contenir deux détecteurs supplémentaires : un dosimètre pour la détection des neutrons de type CR-39 et un dispositif appelé Imaging permettant de déterminer les conditions d'exposition d'un dosimètre (statique, dynamique ou due à une contamination). 


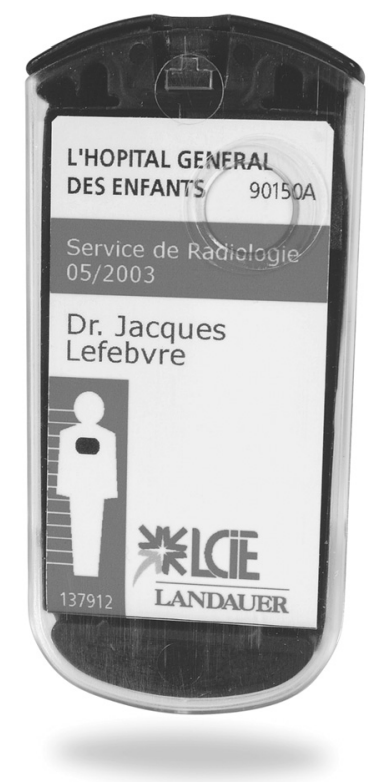

Figure 2 - Le dosimètre InLight ${ }^{\circledR}$.
The InLight ${ }^{\circledR}$ dosimeter.

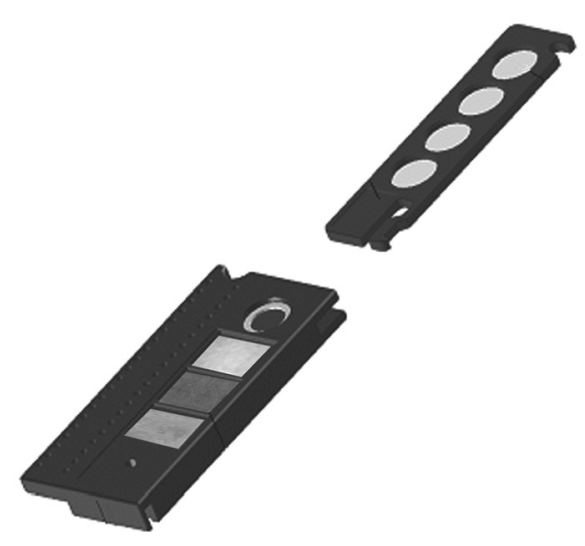

Figure 3 - Le porte-écrans InLight ${ }^{\circledR}$.

The InLight ${ }^{\circledR}$ case.

\subsection{Les lecteurs}

L'analyse d'un InLight ${ }^{\circledR}$ s'effectue à partir de lecteurs qui ont été spécialement adaptés à la technologie OSL. Ces lecteurs utilisent des diodes électroluminescentes comme source lumineuse et sont capables de lire automatiquement un dosimètre en 10 secondes. L'information contenue dans les quatre détecteurs 
est analysée en fonction du facteur de sensibilité mentionné sur la carte du dosimètre, du facteur d'étalonnage du lecteur et des algorithmes de calcul inclus dans le logiciel du lecteur, à savoir pour une mesure en $\mathrm{H}_{\mathrm{p}}(10)$ et en $\mathrm{H}_{\mathrm{p}}(0,07)$.

Quatre modèles de lecteurs sont opérationnels : un lecteur capable de traiter jusqu'à 500 dosimètres, un autre jusqu'à 200 dosimètres, un lecteur manuel et un lecteur portable. Ce dernier est particulièrement adapté pour des applications sur site où des lectures peuvent être nécessaires.

\section{Conclusion}

L'introduction d'un nouveau dosimètre passif basé sur la combinaison technologie OSL et oxyde d'aluminium offrent de nouvelles perspectives pour la surveillance dosimétrique externe des travailleurs susceptibles d'être exposés aux rayonnements ionisants. Seuil de sensibilité plus bas, détecteurs plus sensibles, relecture possible des dosimètres, procédé de fabrication et de traitement simplifié, différenciation des rayonnements $\mathrm{X}$, gamma et bêta en sont les principaux avantages.

\section{Pour en savoir plus}

Botter-Jensen L., McKeever S.W.S., Wintle A.G. (2003) Optically Stimulated Luminescence Dosimetry. Elsevier.

Perks C.A., LeRoy G., Yoder C., Passmore C. (2004) Development of the InLight Monitoring Service for World-wide Application, In Proc 11th IRPA Congress, Madrid, May. 